This item was submitted to Loughborough's Research Repository by the author.

Items in Figshare are protected by copyright, with all rights reserved, unless otherwise indicated.

\title{
Guided recovery: An interpretative phenomenological analysis of service users' experiences of guided self-help for bulimic and binge eating disorders
}

\section{PLEASE CITE THE PUBLISHED VERSION}

https://doi.org/10.1016/j.cbpra.2017.08.004

\section{PUBLISHER}

(c) Association for Behavioral and Cognitive Therapies. Published by Elsevier Ltd

\section{VERSION}

AM (Accepted Manuscript)

\section{PUBLISHER STATEMENT}

This paper was accepted for publication in the journal Cognitive and Behavioral Practice and the definitive published version is available at https://doi.org/10.1016/j.cbpra.2017.08.004.

LICENCE

CC BY-NC-ND 4.0

\section{REPOSITORY RECORD}

Plateau, Carolyn, F.A. Brookes, and M. Pugh. 2019. "Guided Recovery: An Interpretative Phenomenological Analysis of Service Users' Experiences of Guided Self-help for Bulimic and Binge Eating Disorders". figshare. https://hdl.handle.net/2134/26301. 
Guided Recovery: An Interpretative Phenomenological Analysis of Service Users' Experiences of Guided Self-Help for Bulimic and Binge Eating Disorders.

\author{
Plateau, C.R., $\mathrm{PhD}^{1 *}$ \\ Brookes, F.A., BSc ${ }^{1,2}$ \\ Pugh, M. DClinPsy ${ }^{2}$
}

${ }^{1}$ National Centre for Sport and Exercise Medicine, School of Sport, Exercise and Health Sciences, Loughborough University, Loughborough, LE11 3TU , UK.

${ }^{2}$ Vincent Square Eating Disorders Service, Kensington \& Chelsea, London, SW10 9NG

*Corresponding author: Carolyn Plateau, National Centre for Sport and Exercise Medicine, School of Sport, Exercise and Health Sciences, Loughborough University, Loughborough, LE11 3TU. Phone: (+44) 1509228487 Email: C.R.Plateau@lboro.ac.uk 


\begin{abstract}
The efficacy of Cognitive Behavioral Therapy-based Guided Self-Help for mild to moderate bulimia and binge eating disorders has been well supported. However, limited research has explored in-depth individual experiences of this treatment approach. In depth semi-structured interviews were conducted with four individuals who had completed Cognitive Behavioral Therapy based Guided Self Help (CBT-GSH) for bulimic or binge eating disorders. The interviews were transcribed verbatim and subsequently analysed using Interpretative Phenomenological Analysis (IPA). Three superordinate themes emerged: Autonomy and volition; A dynamic relationship: the guided and the guide; and The unwanted friend. The reciprocal nature of the guide/guided relationship was identified as integral to the success of the therapeutic approach. However, participants expressed initial uncertainty towards the therapeutic process, and experienced an uncomfortable dissonance between a lack of volition in therapy seeking and the need to continually self-prescribe CBT-GSH. The findings affirm the central role of the guide in promoting motivation to engage with therapy and highlight the potential benefits of in-session weighing. However, it may be necessary to provide additional support on commencing CBT-GSH to address concerns about the therapeutic approach in this group.
\end{abstract}

Keywords: Cognitive behavioral therapy; eating disorder; low intensity interventions; motivation; satisfaction 
Guided Recovery: An Interpretative Phenomenological Analysis of Service Users’ Experiences of Guided Self-Help for Bulimic and Binge Eating Disorders.

Eating disorders are serious mental health conditions that are characterised by disturbances in eating attitudes, behaviours and perceptions of shape and weight. Eating disorders have a lifetime incidence of around 1\% (Hudson, Hiripi, Harrison \& Kessler, 2007), but are most common among female adolescents (Smink, van Hoeken \& Hoek, 2012). Disordered eating symptoms affect up to a third of women at any one time (Reba-Harrelsen et al., 2009), and these symptoms can progress into more severe clinical disorders if left untreated (Stice, Marti \& Durant, 2011).

Within the UK, treatment for eating disorders follows a stepped care approach. Current guidelines recommend evidence-based self-help programmes as a first line of therapy for bulimia nervosa (BN) and binge eating disorder (BED; National Institute for Clinical Excellence (NICE), 2004). If required, patients can be stepped up to receive more intensive therapy, such as Cognitive Behavioural Therapy for Bulimia Nervosa (CBT-BN). Self-help programmes require patients to work through an online or book-based treatment manual stepby-step (Wilson \& Zandberg, 2012). Such programmes often adopt a cognitive-behavioural therapy (CBT) framework, through incorporating elements of psychoeducation and skills training, and reducing psychological distress and eating disorder symptoms by promoting the development of more adaptive cognitions and behaviours (Fenn \& Byrne, 2013). Self-help interventions can be entirely self-directed (pure self-help) or can involve input and support from healthcare professionals (guided self-help).

Guidance in self-help programmes follows Gould’s parameters of, “clarifying procedures, answering questions and providing...support or encouragement” (Gould \& Clum, 1993). Guided self-help (GSH) for bulimic and binge eating disorders can be facilitated by 
non-specialist healthcare providers, and is flexible enough to be delivered in a variety of formats (e.g., online, on the telephone, or face-to-face; Carrard et al., 2011; Palmer, Birchall, McGrain \& Sullivan, 2002). The frequency and duration of guidance sessions is also considerably reduced in comparison to more intense therapies. Traditional CBT-BN can include 16-20 sessions over 4-5 months, whereas CBT-based guided self-help for bulimic and binge eating disorders (CBT-GSH) may only include up to 12 guidance sessions, up to 20 minutes each (NICE, 2004). The flexible nature of CBT-GSH can help to reduce logistical barriers to treatment, which is important for a population that are traditionally reticent towards seeking support (Carrard et al., 2011; Sysko \& Walsh, 2008). A self-help based approach can also help to promote autonomy and empowerment in treatment for BN and BED, due to the participant-led nature of the therapy (Fairburn \& Carter, 1997; McClay, Waters, McHale, Schmidt, \& Williams, 2013).

The advantages and efficacy of CBT-GSH have been widely researched and supported, with evidence to support the efficacy and cost-effectiveness of CBT-GSH for BN and BED (Lynch et al., 2010; Sanchez-Ortiz \& Schmidt, 2010; Sysko \& Walsh, 2008), and particularly for patients with mild to moderate BED (Beintner, Jacobi \& Schmidt, 2014). For example, a recent metaregression demonstrated an overall effect in favour of CBT-GSH compared to controls in reducing eating disorder psychopathology (a moderate effect size equating to half a point on the EDE-Q/EDE was observed) and in reducing binge abstinence (19\% increased likelihood of abstinence for those receiving CBT-GSH; Traviss-Turner, West \& Hill, 2017). Moderators of improvement suggest that abstinence from binge eating is most likely in participants with a BED diagnosis (Traviss-Turner et al., 2017). Current guidelines emphasise the importance of identifying and incorporating patient perspectives into therapeutic approaches in order to successfully treat eating disorders (NICE, 2004; Peterson, Becker, Treasure, Shafran \& Bryant-Waugh, 2016). Indeed, qualitative studies can 
complement outcome-based research, through enhancing our understanding of the participant motives, expectations, concerns and experiences of engaging in therapy. In addition, qualitative research may help to reveal components of CBT-GSH that are more or less acceptable and/or helpful, which could facilitate improvements to delivery and potentially efficacy of the treatment. At present, a small body of qualitative research has attempted to explore the patient experience of self-help programmes, and to identify ways in which they could be improved.

In particular, qualitative research has sought to understand the nature of guidance in GSH eating disorder programmes, and to identify how the guide may contribute towards successful treatment outcomes. Research has suggested that an open, strong and collaborative therapeutic relationship is important for positive treatment outcomes among BN and BED patients (Banasiak et al., 2007; Traviss, Heywood-Everett \& Hill, 2013). Indeed, successful GSH was experienced as a “joint venture” between the patient and the guide, characterised by reciprocity and trust (Traviss et al., 2013). However, some patients were dissatisfied with the amount of guidance offered and the level of expertise of the guide in addressing eating disorder symptoms (Banasiak et al., 2007; McClay et al., 2013). Indeed, participants particularly value guidance that is available frequently and easily, as demonstrated in a recent community survey of individuals with bulimic symptoms (McClay, Waters, Schmidt \& Williams, 2016). Participants reported that the inclusion of regular, responsive, and remotely delivered support were desirable features of web-based self-help programmes for BN and BED (e.g., via text messaging, email or through forums; McClay et al., 2016).

CBT-GSH is appealing to BN and BED patients because it is flexible, accessible and encourages a participant-led approach to therapy (Banasiak et al., 2007; Sanchez-Ortiz et al., 2011a). However, high levels of drop out and low levels of adherence to therapy are also 
apparent (Beintner, Jacobi \& Schmidt, 2014). For example, dropout rates of up to $88 \%$ have been reported (Beintner et al., 2014). In depth qualitative research has revealed that participants often struggle to motivate themselves to engage with self-help programmes due to a perceived lack of support and accountability (Pretorius, Rowlands, Ringwood \& Schmidt, 2010; Sanchez-Ortiz et al., 2011a). Low levels of motivation may also be due to discrepancies between patient expectations of treatment and the treatment provided (Murray et al., 2003; Sanchez-Ortiz et al., 2011a). However, conflicting evidence suggests that CBTGSH for BN and BED may actually enhance participant motivation to engage with therapy (Loeb, Wilson, Gilbert \& Labouvie, 2000). Whilst this has yet to be explored in depth, some participants have reported that a positive and reassuring therapeutic relationship with the guide helped them to negotiate the more challenging aspects of therapy (Banasiak et al., 2007; Loeb et al., 2000; McClay et al., 2013).

As yet, only a small body of qualitative research has explored patient experiences of CBT-GSH, and studies have yet to adopt interpretative methodologies to understand how patients experience CBT-GSH. Interpretative Phenomenological Analysis (IPA) is commonly used within health psychology to facilitate the exploration of the personal meanings that individuals assign to their illness experiences (Brocki \& Wearden, 2006; Smith \& Osborn, 2008). It draws upon phenomenological origins in that it involves a detailed examination of the participant's lived experiences, and is concerned with individual subjective experiences, rather than attempting to formulate objective accounts (Smith, Flowers \& Larkin, 2009). IPA is grounded in hermeneutics; recognising the central role of the researcher in generating interpretive insights, but also acknowledging that interpretations are bounded by the abilities of participants to articulate their experiences (Brocki \& Wearden, 2006; Smith \& Osborn, 2008). IPA aims to go beyond a simple description of the participant 
experience, but also to interpret and contextualise the findings in relation to the social, cultural, and theoretical context (Larkin, Watts \& Clifton, 2006).

The present study adopts interpretative qualitative methodology (IPA) in order to further our understanding of patient experiences of engaging in CBT-GSH for BN and BED. It is anticipated that this will generate information about the therapeutic experience that can subsequently be utilised to improve experiences of CBT-GSH for future service-users. Microcosmically, participating in this study is likely to prompt individual reflection, allowing participants to assemble their success on completion of CBT-GSH.

\section{Method}

\section{Participants}

Participants were a purposive sample of individuals who had completed CBT-GSH at a UK eating disorders service. Participants were invited to participate if they had completed CBT-GSH, no longer met eating disorder diagnostic criteria and were not in active treatment. This was to ensure that an exploration of the full therapeutic journey (from assessment through to completion) was possible within the interviews, and to minimise any distress or discomfort that could have arisen from discussing on-going therapy. Four female participants aged between 23 and 34 years took part $(M d n=29$ yrs $)$. Two participants had a previous diagnosis of $\mathrm{BN}$, one participant had BED, and the final participant had a previous diagnosis of Other Specified Feeding or Eating Disorder (OSFED). Whilst there is no 'right' sample size for IPA studies the inclusion of large sample sizes can result in the loss of potentially subtle inflections of meaning, and as such a more concentrated focus on a small number of cases is usually preferred (Smith et al., 2009). The sample of four cases was therefore deemed to be in line with these requirements, whilst ensuring that the sample remained a relatively homogeneous group for exploring insights into experiences of CBT-GSH. Ethical approval 
for the study was awarded by the local Research and Development committee and NHS ethical advisory committees.

\section{Procedure}

Participants had completed the CBT-based self-help book Overcoming Binge Eating (Fairburn, 2013), during which they had face-to-face individual guidance from an Assistant Psychologist over 12 weeks, meeting once a week for four weeks and twice a month thereafter. Information about the study was provided by their CBT-GSH facilitator on completion of therapy, and interested participants were then contacted by the researcher (second author), who had not been involved with the participant's therapy. Seventeen participants completed therapy during the time frame of the study (October 2014-May 2015) and were provided with information about the study; four expressed an interest and took part. Participants had the opportunity to ask further questions and discuss the study with the researcher prior to agreeing to take part. Interviews were conducted by the second author and lasted between 45 and 60 minutes. All of the interviews took place within one month of treatment completion. The interview schedule was developed following a review of the CBT-GSH literature and in consultation with clinical and academic experts. Guidance on the structure of the interview schedule was sought from published literature (Smith \& Osborn, 2008), and the interview schedule was employed in a flexible and responsive manner, according to participant responses. The schedule included open questions about the participant's experiences of completing CBT-GSH, including their feelings; motivation; relationship with their guide; and helpful and unhelpful aspects of therapy. The interview schedule was used flexibly to ensure the discussion remained focused on the participant experience, in line with the fundamental principles of IPA (Smith et al., 2009). The topics covered within the interview and example questions and prompts are provided in Table 1.

\section{Data Analysis}


The analysis was conducted by the second author, and followed a series of stages in accordance with IPA methodology (Smith et al., 2009). First, the interviews were transcribed verbatim, and the recordings were listened to several times to enhance familiarity with the dataset and to ensure accuracy in the transcript. Identifying information was removed to uphold participant anonymity and confidentiality. Individual transcripts were studied in detail, noting features of significant, as well as conflicting, corroborating and novel information, specifically in relation to CBT-GSH experiences. The analysis and theme selection was driven by the salience and relevance of the responses to participant experiences of CBT-GSH, as opposed to simply the prevalence or frequency of occurrences (Brocki \& Wearden, 2006). Emergent themes were grouped on a case by case basis, which helped to shape initial frameworks, and facilitated the identification of common or contrasting themes across transcripts. Initial transcripts were reassessed for themes that emerged later on in the analysis, and to check that the work remained grounded in the participants' experience. Finally, superordinate themes were formed, evidencing regular patterns and connections. Suitable quotes identified from across the dataset were identified to illustrate the findings. Grounding the findings in examples ensures that the analytic procedures and interpretation are made transparent, and allows the reader to conceptualise possible alternative meanings and understandings (Elliott, Fischer \& Rennie, 1999). The validity and credibility of the analysis was further supported by discussing the analysis periodically with other members of the research team (Smith \& Osborn, 2008). An audit trail of analytic decisions was maintained to ensure transparency of the analytic process. The research team acted as 'critical friends (Smith \& McGannon, 2017), whereby discussions were held at each analytic stage to identify areas of contention or uncertainty, and to provoke alternative interpretations of the data. This process was conducted not in order to reach a consensus, but to encourage reflection upon the analysis, and to identify any potential areas that might have been 
influenced by assumptions (Yardley, 2014). It is an important process for ensuring the credibility of the final account (Smith, Spiers, Simpson \& Nicholls, 2017).

\section{Results}

The analysis revealed three superordinate themes: (1) Autonomy and volition; (2) A dynamic relationship: the guided and the guide; and (3) The unwanted friend.

\section{Autonomy and Volition}

A struggle between the presence of autonomy and the absence of volition was evident among participants when seeking support and in engaging with CBT-GSH. Actively seeking treatment for an eating disorder was contrasted with a perceived lack of choice in this process. The extent of suffering was such that seeking treatment was no longer an option, but became a necessity, as one participant described: "I need to readdress it...it's making me miserable...I hate...that it's always a factor of my life”. Participants continued to experience these tensions when undertaking CBT-GSH, with therapeutic tasks experienced as something that they had to do, despite a lack of external prescription: "I'd have to read a chapter of a book, and I'd have to complete certain tasks and every week I'd have to monitor what I ate".

However, having a sense of control over the delivery and pace of CBT-GSH was important in promoting engagement with therapy, and in contributing towards a positive experience, as one participant described: "I was kind of feeling better when I started to come and... [guide] kind of gave me that freedom of doing it at my own pace”. However, participants also referred to the effortful nature of engaging with the treatment, and emphasised that it was not always a smooth process. "I was allowing myself food and I was trying to be okay with that and I really was battling”.

The eating disorder was often experienced as a distinct entity, separate from the individual. For example, one participant viewed the eating disorder as a thief: “... [the ED has] just taken away from my life really. Indeed, all of the participants consistently 
semantically reduced their eating disorder, preferring to explain their previous eating difficulties using only diagnostic classifications or through referring to their eating disorder as 'it'. However, CBT-GSH encourages and utilises such distinctions, by promoting selfawareness and allowing action that is independent from it. As one participant described, this process can be empowering and motivating:

[CBT-GSH] breaks down each part of the disorder and...creates a lot of selfawareness, things that you didn't realise you were doing, and that awareness just helped motivate me to come back into the sessions so I can learn more about it...myself and the disorder.

Among completers of CBT-GSH, participants experienced an uncomfortable dynamic between the need to continually self-prescribe CBT-GSH, and the perceived absence of volition. However, developing a sense of control over the therapeutic process and enhancing self-awareness and understanding of the eating disorder seem to be important in facilitating motivation to continue with therapy.

\section{A Dynamic Relationship: The Guided and the Guide}

The lived-experience of CBT-GSH was extensively categorised through an individual's relation to others, and in particular to their guide. The reciprocal nature of the relationship with the guide, the personal qualities of the guide and facilitating discussions and disclosure to others were identified as important elements of the success of CBT-GSH. Positivity about CBT-GSH stemmed from the reciprocity between the guide and patient, creating a comfortable session experience “...Like if you were talking to friends, you feel there's a give and take". In particular, meeting face-to-face with the guide was viewed as integral to the success of therapy and the resolution of their eating issues: "I wouldn't have done as well. I don't think I would have got the same outcome, I don't think I'd be like, as good as I am now if I didn't have [guide]...The same participant described how the close 
relationship with the guide developed through face-to-face meetings was integral to reducing feelings of shame and embarrassment around the eating disorder, and in encouraging greater openness and honesty during CBT-GSH.

"I think with face to face [support] you get to build a better rapport with who you're working with. With eating disorders especially, you do feel embarrassed, and once you kind of get over that you can focus more on your tasks and being more open and honest”.

For some, face-to-face contact and discussion was perceived to be an intrinsic part of therapy: "I came here because I wanted to talk to someone" However, all of the participants valued and enjoyed working with their guide in order to resolve their issues, and reflected upon the importance of the guide's nature in enhancing their experiences of therapy. In particular, guides were identified as patient, honest, supportive and non-judgemental; qualities which were identified as facilitating the development of a positive rapport. "I think [guide is] very patient, non-judgemental, so straight from the beginning, um, yeah she built a good rapport so, I think that makes a big difference... I trusted what she said and she was very patient." The guide was also perceived to support the participants beyond the call of duty, and was viewed as a respected equal, rather than dominating or controlling, which contributed towards the positive experiences of the participants. One participant described:

[guide] was really supportive...she was on my side, anything that I needed, she was going to try to sort out, you know if I had another few questions [guide] was very good about following that up...she was definitely on my side...trying to work with me, for me.

In contrast, the guide held a dominant position as the weigher. However, submission was volitional, with the governing figure assisting, rather than forcing. Indeed, the role of the 
guide in weighing was perceived as helpful, particularly in providing support during a stressful experience, and in maintaining an appropriate weighing pattern. “ With the weighing, I think it's good having someone there...watching you weigh...I think it can be very stressful, yeah, and having someone there, yeah it takes a bit of the stress away". Recognising and accepting the guide's dominant position as the weigher had a positive impact on the participant's day-to-day experience, by promoting self-awareness and reducing their eating disorder symptoms, as one participant describes: “It's definitely made me aware of how much I weigh myself cause... if I did weigh myself, I was like oh s***, I'll have to tell [guide] I've weighed myself...I know I'm not meant to”. For some participants, allowing the guide control as the weigher can help to remove a troubling ED experience, and enhance selfawareness of their eating disorder symptoms.

In addition, disclosure in the guide/guided relationship facilitated disclosure to others over time. This helped the participants to establish an external support-network that helped to actively counter ED symptomology. As one participant described:

At the beginning, I was just doing it by myself... but then I found out one of my work colleagues has exactly the same thing and that just kind of took a bit of weight off me knowing that I could like just talk to her about little things that people without an eating disorder would not understand and so that definitely helped.

Disclosure ended an exhausting process, indicating how the CBT-GSH experience can promote and maintain recovery. However, some participants did not receive the level of support and/or understanding that they had hoped for: “I can't really talk to my friends as much as I'd like to because they all say oh you're fine...I overeat too", and as a result advocated for the inclusion of group sessions into therapy to allow them to share their experiences and to extend their support network to include like-minded others. 


\section{The Unwanted Friend}

The final superordinate theme highlights how perspectives and experiences of CBTGSH changed over the course of the therapy. Participants described being initially sceptical about the value of CBT-GSH; however over time the self-help book gained status as an independent helper. Acceptance of the therapeutic process allowed the book to transcend, acting as a source of reassurance and barrier to potential relapse.

Participant's initial thoughts about CBT-GSH and using the book in therapy contrasted with the largely positive CBT-GSH experience. Participants noted that they were concerned over the legitimacy of the therapy, perhaps due to a lack of familiarity with the concept, and expressed beliefs that they were being offered a 'second best' treatment. On completion, this was no longer perceived to be the case, as one participant described: When they mentioned it was, like the idea of following a book, I kind of thought, oh I don't know about this. I know it sounds a bit silly, but like when they used to talk about the waiting times and they said look if you need CBT, we'll put you on the list for it but right now there's this programme and so, in my head automatically I just thought well it's probably second best, but I don't think that now.

However, being familiar with the general therapeutic context was beneficial in promoting self-efficacy and engagement with CBT-GSH. Specifically, familiarity with CBT helped to validate the book, countering initial uncertainties and helping to build positive foundations. "I kind of knew the process of sort of CBT so, I always was positive because I knew that each week I'd be feeling better".

The participants described being pleasantly surprised with the CBT-GSH process, particularly with regards to the discovery of new information, which served to validate CBTGSH as both an interesting and legitimate therapeutic experience. "I was kind of sceptical at 
the beginning and... when I arrived I started to discover new things and it became more interesting...I realised that I could really like get something out of it”. Participants described how the information within the book helped to challenge previous misconceptions and beliefs, as well as helping to reduce their eating disorder symptoms. This had an extensive impact on the experience and wellbeing of the participants, as one participant described: " $I$ thought that I knew...pretty much everything about it [ED] but yeah, turns out I didn't and that new information was enough to stop me vomiting, so yeah I think that was really helpful”.

The book became personified as an independent helper that the participants trusted and relied upon. It was a source of reassurance, and was identified as a constant that would exist beyond the completion of the face to face therapy sessions. "It's not like it's finished, yeah technically you've still got the book there to help”.

\section{Discussion}

The study aimed to explore the thoughts, experiences and opinions of completers of CBT-GSH. Three superordinate themes were identified, providing an insight into participant experiences of completing CBT-GSH: Autonomy and volition; A dynamic relationship: The guided and the guide; and The unwanted friend. Participant experiences of CBT-GSH were epitomised by acceptance of the therapy and an enhanced insight into their disorder. Supportive and reciprocal relationships with the CBT-GSH guides helped to promote motivation to engage with the therapeutic approach and in creating a comfortable CBT-GSH atmosphere. However, participants also experienced some tensions and difficulties in engaging with CBT-GSH. There was an uncomfortable dissonance between a lack of volition in therapy seeking and the need to continually self-prescribe CBT-GSH, in order to overcome the eating disorder. In addition, participants reported that they were initially unconvinced over the efficacy and usefulness of the approach. However, subsequent 
engagement altered these beliefs through validation from the efficacy of the treatment, acquiring new information, and the guide/patient relationship. As CBT-GSH was accepted, it became inherently motivating and interesting for individuals.

The findings suggest that for some individuals, the beginning of CBT-GSH may be a vulnerable period, where motivation is low for patients with bulimic and binge eating disorders. Initial uptake and engagement can be effortful and uncomfortable, even when guidance is being provided face-to-face, and on a weekly basis. The results of this study suggest that this could partially be due to a lack of familiarity and understanding of CBTGSH, and concerns over the legitimacy of the therapeutic approach. These findings complement results from recent randomized controlled trials (RCTs), which identify low perceived treatment credibility and expected usefulness as predictors of non-take up and drop out from CBT-GSH interventions for BN (Murray et al., 2003; Watson et al., 2016). Treatment seeking is particularly challenging for individuals with BN and BED, due to the perceived shame and stigma associated with bulimic behaviours (Hepworth \& Paxton, 2007), and retaining participants in therapy can also be challenging (Watson et al., 2016). Additional information provision about CBT-GSH at the start of therapy may therefore be beneficial to validate the therapy further and to lessen initial concerns and misconceptions. The acceptability of using a self-help book for mental health concerns, and specifically for eating disorders, should also be promoted. Schemes in the UK such as the Books on Prescription (BOP) service may help to advance acceptability and reduce stigma (The Reading Agency, 2015). BOP allows General Practitioners to recommend a self-help book for individuals with mild-moderate mental health issues. Individuals can borrow these books free of charge from public libraries in the UK (The Reading Agency, 2015).

However, the findings also indicate that participants found CBT-GSH inherently motivating, and in particular that motivation could be enhanced through positive interactions 
with the guide. Indeed, the CBT-GSH facilitators had a key role in enhancing empowerment, autonomy and motivation among participants. This is supported by findings from Banasiak and colleagues (2007) and Traviss and colleagues (2013), who revealed the centrality of a positive therapeutic alliance in promoting successful outcomes in CBT-GSH. However, the findings from the present study explore this concept further, and identify how the guide/patient relationship can facilitate recovery through a dynamic sense of teamwork. This partnership may help to break the negative dyadic relationship between the ED and the self. The guide’s qualities further promoted engagement and recovery, principally through advancing a sense of security. In addition, the findings point towards the therapeutic value of certain aspects of CBT-GSH techniques, notably in-session weight checking (conducted by the guide), and also in promoting self-awareness and independence from the eating disorder. For some participants, allowing the guide control as the weigher can enhance self-awareness and consequently, help to reduce their ED symptoms. Weight checking is recognised as a key intervention in CBT for eating disorders (Waller \& Mountford, 2015) but is not used across all GSH programmes (e.g., Treasure et al., 1994). Further studies would help confirm this finding and determine whether in-session weight checking enhances the effectiveness of GSH interventions. Similarly, the experience and conceptualisation of the ED as a distinct entity to the self is not unique to the guided self-help context for bulimic and binge eating disorders (e.g., Smith et al., 2016), but it does suggest that enhanced self-awareness of the eating disorder as developed through the CBT-GSH process may be helpful in promoting a positive therapeutic outcome.

Whilst the importance of face-to-face meetings with the guide was highlighted as critical for the success of the participants in this study, a number of alternative CBT-GSH delivery methods have been explored, for example, via telephone (Wells et al., 1997) or via the internet (Pretorius et al., 2010). Such approaches are attractive due to greater potential 
cost-effectiveness, and evidence suggests that these alternative delivery methods are acceptable and feasible for delivery in this population (e.g., Carrard et al., 2011; SanchezOrtiz et al., 2011b). Recent evidence from McClay et al., (2016) indicated that online GSH programmes for bulimia were popular among a community sample of individuals presenting with bulimic symptoms, and the flexibility and regularity of support provided may be more important than offering guidance face-to-face. Indeed, for some, face-to-face contact is undesirable (McClay et al., 2013), with evidence to suggest that younger participants may be more comfortable in communicating and seeking support online (Kauer, Mangan \& Sanci, 2014). Further in-depth research with those who have taken part in online CBT-GSH may offer additional important insights into the nature of the guide/patient relationship in these alternative therapeutic contexts. Recognising and accommodating (where possible) different preferences for the delivery mode of therapy could, however, be an important way to reduce drop out and improve engagement (Watson et al., 2016). It is acknowledged however, that there is a need to effectively manage costs and demand.

The participants in this study did not raise any contentious issues with regards to the overall suitability of CBT-GSH as a low-intensity treatment for BN and BED. CBT-GSH was perceived to be an effective and satisfying treatment that can be a valuable resource for services, particularly in challenging economic circumstances (Sysko \& Walsh, 2008). However, it is imperative to acknowledge that a lack of contention does not necessarily equate to a viable proposition, and the limitations of this study need to be considered. Importantly, the participants in the study had all successfully completed CBT-GSH, so it is not surprising that their experiences were positive. The sample size was small; however the focus is predominantly on the individual experience in idiographic qualitative research and larger sample sizes can lead to a loss of potentially subtle inflections of meaning (Brocki \& Wearden, 2006). However, it is important to acknowledge that the findings from this small 
sample of participants may not represent the experiences of the wider population undergoing treatment for CBT-GSH. In addition, the participants in this study were all female, aged between 23-34 years, and had all taken part in a form of CBT-GSH that involved regular, face-to-face meetings with a guide. Additional interpretative research with individuals who chose not to start therapy, did not complete CBT-GSH, or who were stepped up to more intensive therapy will likely reveal further nuances and different ways in which the therapy could be improved, and this is an important next step towards refining and developing appropriate guided self-help programmes. Incorporating perspectives of non-completers would provide a wider understanding of patient experiences within the context of CBT-GSH. In addition, all the participants in this study worked through the same GSH protocol. There are other evidence-based GSH protocols that adopt different approaches (e.g., Cooper, 2009; Schmidt \& Treasure, 2009). The findings from this study are likely to be specific to the protocol utilised, and further exploration of the experiences of those working through alternative GSH protocols is warranted.

The results of this study can also help to inform clinical practice. Specifically, the results affirm the significant role of the guide in promoting motivation to engage with therapy, and in creating a comfortable and positive therapeutic atmosphere. However, the guide may need reinforce and/or provide additional information at the start of therapy in order to alleviate patient concerns over the usefulness of the CBT-GSH approach. The findings also suggest that in-session weighing is particularly valuable for ED patients undergoing CBT-GSH. This finding needs to be confirmed with larger scale studies, and research is needed to explore the potential impact of in-session weighing on efficacy of CBTGSH in this group. In addition, further exploration of how the guide's nature and the guide/patient relationship could impact on engagement and completion of treatment would 
provide useful information for future training of CBT-GSH facilitators. Further research incorporating the experiences and perspectives of guides will help to illuminate this issue.

In summary, this study is novel in its in-depth exploration of individual experiences and perceptions of the CBT-GSH for BN and BED. It offers important insights into the key elements of CBT-GSH that can facilitate successful experiences among BN and BED patients; notably the central role of the guide in promoting motivation to engage with therapy and the value of in-session weighing. The findings also identify some of the key challenges for BN and BED patients in engaging with CBT-GSH. Participants found it challenging to continually self-prescribe and work through the manual independently, and were initially sceptical about the value of the therapy. The findings suggest a need to provide additional support and information for individuals when commencing CBT-GSH for eating disorders to address concerns and uncertainties about the therapeutic approach. 


\section{References}

Banasiak, S.J., Paxton, S.J., \& Hay, P.J. (2007). Perceptions of cognitive behavioural guided self-help treatment for bulimia nervosa in primary care. Eating Disorders, 15(1), 23-40. Doi: 10.1080/10640260601044444

Beintner, I., Jacobi, C., \& Schmidt, U.H. (2014). Participation and outcome in manualized self-help for bulimia nervosa and binge eating disorder - A systematic review and metaregression analysis. Clinical Psychology Review, 34(2), 158-176. Doi: 10.1016/j.cpr.2014.01.003

Brocki, J.M., \& Wearden, A.J. (2006). A critical evaluation of the use of interpretative phenomenological analysis (IPA) in health psychology. Psychology and Health, 21(1), 87-108. Doi: 10.1080/14768320500230185.

Carrard, I., Crepin, C., Rouget, P., Lam, T., Golay, A., \& Van der Linden, M. (2011). Randomised controlled trial of a guided self-help treatment on the Internet for binge eating disorder. Behaviour Research and Therapy, 49(8), 482-491. Doi: 10.1016/j.brat.2011.05.004

Cooper, P. (2009). Overcoming bulimia nervosa and binge eating: A self-help guide using cognitive behavioral techniques ( $2^{\text {nd }}$ ed.). London: Robinson.

Elliott, R., Fischer, C.T., \& Rennie, D.L. (1999). Evolving guidelines for publication of qualitative research studies in psychology and related fields. British Journal of Clinical Psychology, 38(3), 215-229. Doi: 10.1348/014466599162782

Fairburn, C.G., \& Carter, J.C. (1997). Self-help and guided self-help for eating problems. In: D.M. Garner, P.E. Garfinkel (Eds.) Handbook of treatment for eating disorders $\left(2^{\text {nd }}\right.$ Ed.) Guilford Press, New York. pp. 494-499.

Fairburn, C. G. (2013). Overcoming Binge Eating ( $2^{\text {nd }}$ ed.). New York: Guilford Press. 
Fenn, K., \& Byrne, M. (2013). The key principles of cognitive behavioral therapy. InnovAiT: Education and Inspiration for General Practice, 6(9), 570-585. Doi: $10.1177 / 1755738012471029$

Gould, R. A., \& Clum, G.A. (1993). A meta-analysis of self-help treatment approaches. Clinical Psychology Review, 13(2), 169-86. Doi: 10.1016/0272-7358(93)90039-O

Hepworth, N., \& Paxton, S.J. (2007). Pathways to help-seeking in bulimia nervosa and binge eating problems: A concept mapping approach. International Journal of Eating Disorders, 40(6), 493-504. Doi: 10.1002/eat.20402.

Hudson, J. I., Hiripi, E., Pope, H. G., \& Kessler, R. C. (2007). The prevalence and correlates of eating disorders in the National Comorbidity Survey Replication. Biological Psychiatry, 61(3), 348-358. Doi: 10.1016/j.biopsych.2006.03.040

Kauer, S.D., Mangan, C., \& Sanci, L. (2014). Do online mental health services improve helpseeking for young people? A systematic review. Journal of Medical Internet Research, 16(3), e66. Doi: 10.2196/jmir.3103

Larkin, M., Watts, S., \& Clifton, E. (2006). Giving voice and making sense in interpretative phenomenological analysis. Qualitative Research in Psychology, 3(2), 102-120. Doi: 10.1191/1478088706qp062oa

Loeb, K.L., Wilson, G.T., Gilbert, J.S., \& Labouvie, E. (2000). Guided and unguided selfhelp for binge eating. Behaviour Research and Therapy, 38(3), 259-272. Doi: 10.1016/S0005-7967(99)00041-8.

Lynch, F. L., Striegel-Moore, R. H., Dickerson, J. F., Perrin, N., DeBar, L., Wilson, G. T., \& Kraemer, H. C. (2010). Cost-effectiveness analysis of guided self-help treatment for recurrent binge eating. Journal of Consulting and Clinical Psychology, 78(3), 322-333. Doi: $10.1037 / \mathrm{a} 0018982$. 
McClay, C., Waters, L., McHale, C., Schmidt, U., \& Williams, C. (2013). Online cognitive behavioral therapy for bulimic type disorders, delivered in the community by a nonclinician: Qualitative study. Journal of Medical Internet Research, 15(3), e46. Doi: 10.2196/jmir.2083

McClay, C., Waters, L., Schmidt, U., \& Williams, C. (2016). A survey of attitudes towards computerized self-help for eating disorders within a community-based sample. Behavioural and Cognitive Psychotherapy, 44(1), 65-78. Doi: 10.1017/S1352465814000484.

Murray, K., Pombo-Carril, M.G., Bara-Carril, N., Grover, M., Reid, Y., Langham, C...\& Schmidt, U. (2003). Factors determining uptake of a CD-ROM-based self-help treatment for bulimia: Patient characteristics and subjective appraisals of self-help treatment. European Eating Disorders Review, 11(3), 243-260. Doi: 10.1002/erv.519.

National Institute for Clinical Excellence (NICE). (2004). Eating disorders: core interventions in the treatment and management of anorexia nervosa, bulimia nervosa and related eating disorders. NICE Clinical Guidelines. London: NICE.

Palmer, R. L., Birchall, H., McGrain, L., \& Sullivan, V. (2002). Self-help for bulimic disorders: a randomised controlled trial comparing minimal guidance with face-to-face or telephone guidance. The British Journal of Psychiatry, 181(3), 230-235. Doi: 10.1192/bjp.181.3.230

Peterson, C.B., Becker, C.B., Treasure, J., Shafran, R., \& Bryant-Waugh, R. (2016). The three-legged stool of evidence-based practice in eating disorder treatment: Research, clinical, and patient perspectives. BMC Medicine, 14, 69. Doi: 10.1186/s12916-0160615-5.

Pretorius, N., Rowlands, L., Ringwood, S., \& Schmidt, U. (2010). Young people’s perceptions of and reasons for accessing a web-based cognitive behavioural 
intervention for bulimia nervosa. European Eating Disorders Review, 18(3), 197-206. Doi: $10.1002 /$ erv.985

Reba-Harrelson, L., Von Holle, A., Hamer, R.M., Swann, R., Reyes, M.L., \& Bulik, C.M. (2009). Patterns and prevalence of disordered eating and weight control behaviors in women ages 25-45. Eating and Weight Disorders, 14(4), e190-198.

Sanchez-Ortiz, V.C., House, J., Munro, C., Treasure, J., Startup, H., Williams, C., \& Schmidt, U. (2011a). “A computer isn’t gonna judge you”: A qualitative study of users’ views of an internet-based cognitive behavioural guided self-care treatment package for bulimia nervosa and related disorders. Eating and Weight Disorders, 16(2), e93-e101. Doi: 10.1007/BF03325314.

Sanchez-Ortiz, V.C., Munro, C., Stahl, D., House, J., Startup, H., Treasure, J...\& Schmidt, U. (2011b). A randomised controlled trial of internet-based cognitive behavioral therapy for bulimia nervosa or related disorders in a student population. Psychological Medicine, 41(2), 407-417. Doi: 10.1017/S0033291710000711

Sanchez-Ortiz, V.C., \& Schmidt, U. (2010). Self-help approaches for bulimia nervosa and binge eating disorder. In: C.M. Grilo, J.E. Mitchell (Eds.), The treatment of eating disorders, Guilford Press, New York. pp. 359-371

Schmidt, U., \& Treasure, J. (2009). Getting better bit(e) by bit(e): a survival kit for sufferers of bulimia nervosa and binge eating disorder ( $7^{\text {th }}$ ed.). London: Brunner-Routledge.

Smink, F.R., van Hoeken, D., \& Hoek, H.W. (2012). Epidemiology of eating disorders: Incidence, prevalence and mortality. Current Psychiatry Reports, 14(4), 406-414. Doi: 10.1007/s11920-012-0282-y.Smith, J. A., Flowers, P., Larkin, A. (2009). Interpretative Phenomenological Analysis: Theory, Method and Research. London, UK: Sage Publications Ltd. 
Smith, B., \& McGannon, K.R. (2017). Developing rigor in qualitative research: Problems and opportunities within sport and exercise psychology. International Review of Sport and Exercise Psychology Doi: 10.1080/1750984X.2017.1317357

Smith, V., Chouliara, Z., Morris, P.G., Collin, P., Power, K., Yellowlees, A... \& Cook, M. (2016). The experience of specialist inpatient treatment for anorexia nervosa: A qualitative study from adult patients’ perspectives. Journal of Health Psychology, 21(1), 16-27. Doi: 10.1177/1359105313520336.

Smith, J. A., Flowers, P., Larkin, A. (2009). Interpretative Phenomenological Analysis: Theory, Method and Research. London, UK: Sage Publications Ltd.

Smith, J. A., \& Osborn, M. (2008). Interpretative Phenomenological Analysis. In J. A. Smith (Ed), Qualitative Psychology: A Practical Guide to Research Methods (2 ${ }^{\text {nd }}$ ed.) (pp. 53 - 81). London, UK: Sage Publications Ltd.

Smith, J.A., Spiers, J., Simpson, P., \& Nicholls. A.R. (2017). The psychological challenges of living with an ileostomy: An interpretative phenomenological analysis. Health Psychology 36(2), 143-151. Doi: 10.1037/hea0000427

Stice, E., Marti, C.N., Durant, S. (2011). Risk factors for onset of eating disorders: Evidence of multiple risk pathways from an 8-year prospective study. Behaviour Research and Therapy, 49(10), 622-627. Doi: 10.1016/j.brat.2011.06.009.

Sysko, R., \& Walsh, B.T. (2008). A critical evaluation of the efficacy of self-help interventions for the treatment of bulimia nervosa and binge eating disorder. International Journal of Eating Disorders, 41(2) 97-112. Doi: 10.1002/eat.20475

The Reading Agency. (2015). Reading well: Books on prescription. Retrieved from: http://reading-well.org.uk 
Traviss, G.D., Heywood-Everett, S., \& Hill, A.J. (2013). Understanding the 'guide' in guided self-help for disordered eating: A qualitative process study. Psychology and Psychotherapy, 86(1), 86-104. Doi: 10.1111/j.2044-8341.2011.02049.x

Traviss-Turner, G.D., West, R.M., \& Hill, A.J. (2017). Guided self-help for eating disorders: A systematic review and metaregression. European Eating Disorders Review, 25(3), 148-164. Doi: 10.1002/erv.2507

Treasure, J., Schmidt, U., Troop, N., Tiller, J., Todd., G., Keilen, M., \& Dodge, E. (1994). First step in managing bulimia nervosa: Controlled trial of a therapeutic manual. $B M J$, 308, 686. Doi: 10.1136/bmj.308.6930.686.

Waller, G., \& Mountford, V.A. (2015). Weighing patients within cognitive-behavioral therapy for eating disorders: How, when and why. Behavior Research and Therapy, 70, 1-10. Doi: 10.1016/j.brat.2015.04.004

Watson, H.J., Levine, M.D., Zerwas, S.C., Hamer, R.M., Crosby, R.D., Sprecher, C.S...\& Bulik, C.M. (2016). Predictors of dropout in face-to-face and internet-based cognitive behavioral therapy for bulimia nervosa in a randomized controlled trial. International Journal of Eating Disorders. Doi: 10.1002/eat.22644

Wells, A. M., Garvin, V., Dohm, F. A., \& Striegel-Moore, R. H. (1997). Telephone-based guided self-help for binge eating disorder: a feasibility study. International Journal of Eating Disorders, 21(4), 314-316. Doi: 10.1002/(SICI)1098-108X(1997)21:4<341::AIDEAT6>3.0.CO;2-O

Wilson, G.T., \& Zandberg, L.J. (2012). Cognitive-behavioral guided self-help for eating disorders: Effectiveness and scalability. Clinical Psychology Review, 32(4), 343-357. Doi: 10.1016/j.cpr.2012.03.001 
Yardley, L. (2014). Demonstrating validity in qualitative psychology. In J. A. Smith (Ed.), Qualitative Psychology. A practical guide to research methods (pp. 235-252). London: Sage. 
Table 1: Summary of topics covered within the interview and example questions and prompts

\section{Topics and example questions}

- Demographics; ED diagnosis and treatment seeking.

\section{A: Overall experience of CBT-GSH}

- Can you tell me about a typical CBT-GSH session?

Prompts: Where/who/when? Impact on routine/work/social life? Feelings

before/during/after CBT-GSH sessions? Why?

\section{B: Professionals involved in CBT-GSH}

- Can you tell me about your initial assessment?

Prompts: Feelings towards CBT-GSH? Understanding of what was involved?

Motivation? Improvements?

- How did your guide make you feel during CBT-GSH?

Prompts: Towards ED? Towards therapy? Impact of guide on overall experience?

Motivation? Helpful/unhelpful features? Improvements/changes?

- How did you find the face-to-face contact in CBT-GSH?

Prompts: Rapport? Logistics? Additional/alternative contact?

\section{C: Resources and delivery method of CBT-GSH}

- How did you find using the manual for CBT-GSH?

Prompts: Relevance? Frequency of use during therapy/afterwards? Impact on motivation/overall experience?

- In what other ways could CBT-GSH have been delivered?

Prompts: Location/setting of sessions? Length of sessions, length of treatment?

Preferences? Impact on experience?

\section{D: Motivation}

- What motivated you to seek treatment initially?

Prompts: How motivated were you to complete CBT-GSH? Change in motivation? Why/when? Readiness/preparedness? Factors that affected motivation?

- How did you find completing the homework tasks?

Prompts: Did you always complete homework? Why/Why not? Feelings towards keeping a food diary? Motivation?

\section{E: Recommendations}

- Is there anything that could have improved your experiences of CBT-GSH?

- What recommendations would you give to professionals working in CBT-GSH and why? 